\title{
CALCULATION OF THE MATTER PROPAGATION IN THE RIVER OR OPEN CHANNEL SYSTEM
}

\author{
Tran Gia Lich \\ Institute of Mathematics, NCST of Vietnam
}

\begin{abstract}
In this paper we consider the following problems: The existence of solution,
\end{abstract} the stability of finite difference scheme and the non-negative property of numerical solution.

\section{Introduction}

The differential equation describing diffusion process of matter $S$ in a river or open channel is as follows:

$$
\frac{\partial S}{\partial t}+v \frac{\partial S}{\partial x}-\frac{1}{\omega} \frac{\partial}{\partial x}\left(\omega P \frac{\partial S}{\partial x}\right)+b S=\phi
$$

where

$x$ is the coordinate along the streamed,

$t$ - the time, $v$ - the average velocity,

$\omega$ - the area of cross section,

$P \geq \delta_{0}>0$ - the general diffusion coefficient,

$b \geq 0$ - the decay coefficient.

Equation (0.1) is of parabolic type. In order to solve this boundary problem, beside the initial condition at the time $t=0: S(x, 0)=S^{0}(x)$, one more boundary condition is needed at every boundary $S\left(L_{i}, t\right)=S_{L_{i}}(t),\left(L_{1}=x_{1}=0, L_{2}=x_{N}=\right.$ $L)$.

At the inflow boundary $L_{i}$ the boundary condition will be given by $S\left(L_{i}, t\right)=$ $f_{i}(t) \geq 0$. At the outflow boundary we will consider that $S\left(L_{i}, t\right)$ is resulted from the transport process or $\left.\frac{\partial S}{\partial x}\right|_{L_{i}}=0$.

In the river or open channel system (see fig. 1) beside the boundary conditions at the boundary nodes $A, B, C$, it is necessary to give the adjoint conditions at the internal nodes $D, E, F$.

These adjoint conditions are resulted from the law of matter conservation and on the supposion that there are no source, no creation, no decay of matter $S$ at the internal nodes (see [5]).

$$
\frac{\partial}{\partial t} \int_{\widetilde{V}} S_{i} d \widetilde{V}+\int_{\widetilde{S}} S_{i} v_{n} d \widetilde{S}+\int_{\widetilde{S}} P_{i}\left(\frac{\partial S}{\partial x}\right)_{i} d \dot{S}=0,
$$




$$
\frac{\partial}{\partial t} \int_{\widetilde{V}} S_{i} d \widetilde{V}+\sum_{i=1}^{I^{D}} \alpha_{i} S_{i} Q_{i}+\sum_{i=1}^{I^{D}} \alpha_{i} \omega_{i} P_{i}\left(\frac{\partial S}{\partial x}\right)_{i}=0
$$

where

$I^{D}$ is the set of branches having common internal node $D$,

$Q_{i}$ - the discharge at the node $D$ of the river branch $i$,

$v_{n}$ - the projection of the velocity vector on the external normal vector of the boundary $\widetilde{S}$,

$$
\alpha_{i}= \begin{cases}1 & \text { if } D \text { is right boundary of river branch } i \\ -1 & \text { if } D \text { is left boundary of river branch. } i\end{cases}
$$

Suppose that the component $\frac{\partial}{\partial t} \int_{\widetilde{V}} S_{i} d \widetilde{V}$ is very small in comparison with the other ones, and the value $S_{i}^{D}$ of the all branches $i\left(i \in I^{D}\right)$ are equal $\left(S_{i}^{D}=S^{D}\right)$ then we obtain the following adjoint condition

$$
\sum_{i=1}^{I^{D}} \alpha_{i} \omega_{i} P_{i}\left(\frac{\partial S}{\partial x}\right)_{i}=0 .
$$

If the diffusion coefficient $P$ does not depend on $S$, the equation (0.1) is linear. In the opposite cases (0.1) is nonlinear equation and we can linearise $(0.1)$, considering $S$ on the coefficient $P$ as known, using the values $S^{k}$ at the previous time step $t_{k}$.

Equation (0.1) can be written in the following form

$$
\frac{\partial S}{\partial t}+a \frac{\partial S}{\partial x}-\frac{\partial}{\partial x}\left(P \frac{\partial S}{\partial x}\right)+b S=\phi
$$

where

$$
a=v-\frac{P}{\omega} \frac{\partial \omega}{\partial x}, \quad P \geq \delta_{0}>0
$$

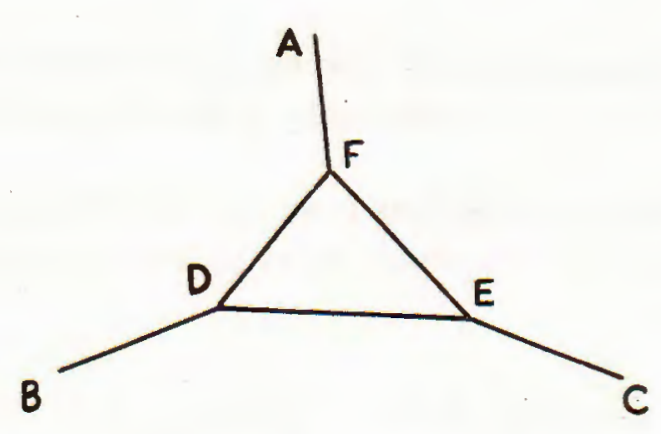

Fig. 1 


\section{Existence of the solution}

The linear equation (0.3) with the initial condition $S(x, 0)=S^{0}(x)$ and the boundary conditions $S\left(L_{i}, t\right)=S_{L_{i}}(t)(i=1,2)$ has a unique solution, if these conditions and the coefficients of the equation (0.3) are sufficiently smooth.

By the transformation

$$
S=C e^{g(x, t)+M t}, \quad M=\text { const }
$$

we have

$$
\begin{aligned}
\frac{\partial S}{\partial t} & =e^{g+M t}\left[\frac{\partial C}{\partial t}+\left(g_{t}^{\prime}+M\right) C\right] \\
\frac{\partial S}{\partial x} & =e^{g+M t}\left(\frac{\partial C}{\partial x}+g_{x}^{\prime} C\right) \\
\frac{\partial}{\partial x}\left(P \frac{\partial S}{\partial x}\right) & =e^{g+M t}\left[\frac{\partial}{\partial x}\left(P \frac{\partial C}{\partial x}\right)+2 P g_{x}^{\prime} \frac{\partial C}{\partial x}+C \frac{\partial}{\partial x}\left(P g_{x}^{\prime}\right)+P C\left(g_{x}^{\prime}\right)^{2}\right]
\end{aligned}
$$

Putting (1.1) into (0.3) yields:

$$
\frac{\partial C}{\partial t}+\left(a-2 P g_{x}^{\prime}\right) \frac{\partial C}{\partial x}-\frac{\partial}{\partial x}\left(P \frac{\partial C}{\partial x}\right)+\alpha C=\widetilde{\phi}
$$

where

$$
\left\{\begin{array}{l}
\alpha=b+M+g_{t}^{\prime}+a g_{x}^{\prime}-\frac{\partial}{\partial x}\left(P g_{x}^{\prime}\right)-P\left(g_{x}^{\prime}\right)^{2} \\
\tilde{\phi}=\phi e^{-g(x, t)-M t}
\end{array}\right.
$$

The function $g(x, t)$ will be chosen so that $a-2 P g_{x}^{\prime}=0$. For this $g_{x}^{\prime}=\frac{a}{2 P}$ or $g(x, t)=\int \frac{a}{2 P} d x$ and $M$ will be chosen so that $\alpha \geq 0$. Then equation (1.2) becomes

$$
\frac{\partial C}{\partial t}=\frac{\partial}{\partial x}\left(P \frac{\partial C}{\partial x}\right)-\alpha C+\tilde{\phi}
$$

with the following initial and boundary conditions

$$
\begin{aligned}
C(x, 0) & =C^{0}(x)=S^{0}(x) e^{-g(x, 0)}, \\
C\left(L_{i}, t\right) & =C_{L_{\mathbf{i}}}(t)=S_{L_{i}}(t) e^{-g\left(L_{i}, t\right)-M t} .
\end{aligned}
$$

We use new transformation

$$
\bar{S}=C-\frac{L_{2}-x}{L_{2}-L_{1}} C_{L_{1}}(t)-\frac{x-L_{1}}{L_{2}-L_{1}} C_{L_{2}}(t)
$$


Putting (1.4) into (1.3) we get:

$$
\frac{\partial \bar{S}}{\partial t}=\frac{\partial}{\partial x}\left(P \frac{\partial \bar{S}}{\partial x}\right)-\alpha \bar{S}+\bar{\phi}
$$

where

$$
\begin{aligned}
\bar{\phi}= & \tilde{\phi}-\alpha\left[\frac{L_{2}-x}{L_{2}-L_{1}} C_{L_{1}}(t)+\frac{x-L_{1}}{L_{2}-L_{1}} C_{L_{2}}(t)\right] \\
& +\frac{C_{L_{2}}(t)-C_{L_{1}}(t)}{L_{2}-L_{1}} \frac{\partial P}{\partial x}-\frac{L_{2}-x}{L_{2}-L_{1}} C_{L_{1}}^{\prime}(t)-\frac{x-L_{1}}{L_{2}-L_{1}} C_{L_{2}}^{\prime}(t)
\end{aligned}
$$

The corresponding initial and boundary conditions of the equation (1.5) are:

$$
\begin{gathered}
\bar{S}(x, 0)=e^{-g(x, 0)}\left(S^{0}(x)-\frac{L_{2}-x}{L_{2}-L_{1}} S_{L_{1}}(0)-\frac{x-L_{1}}{L_{2}-L_{1}} S_{L_{2}}(0)\right) \\
\bar{S}\left(L_{i}, t\right)=0 ; \quad(i=1,2) .
\end{gathered}
$$

The boundary problem (1.5), (1.6) with the coefficient $P \geq \delta_{0}>0$ and $\alpha \geq 0$. has a unique solution (see [4]). Therefore the linear equation (0.3) has also a unique solution.

\section{Stability of the finite difference scheme}

Differencing the equation (0.1) we get:

$$
\begin{aligned}
& \frac{S_{n}^{k+1}-S_{n}^{k}}{\tau}+\frac{(v+|v|)_{n}}{2} \frac{S_{n}^{k+1}-S_{n-1}^{k+1}}{\Delta}+\frac{(v-|v|)_{n}}{2} \frac{S_{n+1}^{k+1}-S_{n}^{k+1}}{\Delta}- \\
& \frac{1}{\omega_{n} \Delta}\left[(\omega P)_{n+1 / 2} \frac{S_{n+1}^{k+1}-S_{n}^{k+1}}{\Delta}-(\omega P)_{n-1 / 2} \frac{S_{n}^{k+1}-S_{n-1}^{k+1}}{\Delta}\right]+b_{n} S_{n}^{k+1}=\phi_{n}^{k+1},
\end{aligned}
$$

where $\Delta=x_{n+1}-x_{n}=$ const, $\tau=t_{k+1}-t_{k}=$ const.

The equation $(2,1)$ can be rewritten under the form

$$
\alpha_{n} S_{n+1}^{k+1}+\beta_{n} S_{n}^{k+1}+\gamma_{n} S_{n-1}^{k+1}=\delta_{n}
$$

where

$$
\begin{aligned}
& \alpha_{n}=-\frac{\tau(|v|-v)_{n}}{2 \Delta}-\frac{\tau(\omega P)_{n+1 / 2}}{\omega_{n} \Delta^{2}} \\
& \beta_{n}=1+\tau b_{n}+\frac{\tau|v|_{n}}{\Delta}+\frac{\tau(\omega P)_{n+1 / 2}}{\omega_{n} \Delta^{2}}+\frac{\tau(\omega P)_{n-1 / 2}}{\omega_{n} \Delta^{2}} \\
& \gamma_{n}=-\frac{\tau(|v|+v)_{n}}{2 \Delta}-\frac{\tau(\omega P)_{n-1 / 2}}{\omega_{n} \Delta^{2}} \\
& \delta_{n}=S_{n}^{k}+\tau \phi_{n}^{k+1}
\end{aligned}
$$


Obviously, the coefficients $\alpha_{n}, \beta_{n}$ and $\gamma_{n}$ satisfy the following conditions

$$
\begin{gathered}
\alpha_{n}<0, \quad \beta_{n}>0, \quad \gamma_{n}<0, \\
\beta_{n}=\left|\alpha_{n}\right|+\left|\gamma_{n}\right|+\sigma_{n}, \quad \sigma_{n}=1+\tau b_{n}>0 .
\end{gathered}
$$

In the simple case when $v=$ const, $\omega=$ const, $P=$ const, $b=$ const, (i.e. $\alpha_{n}=\alpha=$ const, $\beta_{n}=\beta=$ const and $\gamma_{n}=\gamma=$ const) we can prove the stability of this finite difference scheme (2.1).

Indeed, substituting the solution under the form $S_{n}^{k}=\lambda^{k} S^{0} e^{i n \varphi}$, where $i=\sqrt{-1}$ and $0 \leq \varphi<2 \pi$ into the equation (2.2) with $\phi=0$ we get

$$
\lambda^{k+1}\left(\alpha e^{i(n+1) \varphi}+\beta e^{i n \varphi}+\gamma e^{i(n-1) \varphi}\right) S^{0}=\lambda^{k} S^{0} e^{i n \varphi} .
$$

Dividing both sides of this expression by $S^{0} e^{i n \varphi}$ one deduces

$$
\begin{aligned}
& \lambda^{k+1}\left(\alpha e^{i \varphi}+\beta+\gamma e^{-i \varphi}\right)=\lambda^{k}, \\
& \lambda^{k+1}[\beta+(\alpha+\gamma) \cos \varphi+i(\alpha-\gamma) \sin \varphi]=\lambda^{k} .
\end{aligned}
$$

From inequality $|(\alpha+\gamma) \cos \varphi| \leq|\alpha+\gamma| \leq|\alpha|+|\gamma|$ it is easy to verify that $\beta+(\alpha+$ $\gamma) \cos \varphi=1+\tau b+|\gamma|+|\alpha|+(\alpha+\gamma) \cos \varphi>1$, therefore

$$
|\lambda|=\left|\frac{\lambda^{k+1}}{\lambda^{k}}\right|=\frac{1}{\sqrt{[\beta+(\alpha+\gamma) \cos \varphi]^{2}+(\alpha-\gamma)^{2} \sin ^{2} \varphi}}<1 .
$$

Let $\left|S_{n_{0}}^{k+1}\right|=\sup _{n}\left|S_{n}^{k+1}\right|$, from (2.2), (2.3) we have

$$
\begin{aligned}
\left|\beta S_{n_{0}}^{k+1}-\right| \alpha\left|S_{n_{0}+1}^{k+1}-\right| \gamma\left|S_{n_{0}-1}^{k+1}\right| & =\left|S_{n_{0}}^{k}+\tau \phi_{n_{0}}^{k+1}\right|, \\
\left|S_{n_{0}}^{k+1}\right|(\beta-|\alpha|-|\gamma|) & \leq \sup _{2 \leq n \leq N-1}\left|S_{n}^{k}\right|+\tau \sup _{k} \sup _{2 \leq n \leq N-1}\left|\phi_{n}^{k}\right|, \\
\sup _{2 \leq n \leq N-1}\left|S_{n}^{k+1}\right| & \leq \frac{1}{1+\tau b}\left[\sup _{2 \leq n \leq N-1}\left|S_{n}^{k}\right|+\tau \sup _{k} \sup _{2 \leq n \leq N-1}\left|\phi_{n}^{k}\right|\right] \\
& <\sup _{2 \leq n \leq N-1}\left|S_{n}^{k}\right|+\tau \sup _{k} \sup _{2 \leq n \leq N-1}\left|\phi_{n}^{k}\right| \leq \ldots \\
\cdots & \leq \sup _{2 \leq n \leq N-1}\left|S_{n}^{0}\right|+(k+1) \tau \sup _{k} \sup _{2 \leq n \leq N-1}\left|\phi_{n}^{k}\right| .
\end{aligned}
$$

Denoting $\left\|S_{h}\right\|_{h}=\sup _{k} \sup _{1 \leq n \leq N}\left|S_{n}^{k}\right|, \quad\left\|\phi_{h}\right\|_{h}=\sup _{k} \sup _{2 \leq n \leq N-1}\left|\phi_{n}^{k}\right|$, $\left\|S_{h}^{0}\right\|_{h}=\sup _{1 \leq n \leq N}\left|S_{n}^{0}\right|, \quad\left\|S_{L_{i}}\right\|_{h}=\sup _{k}\left|S_{L_{i}}^{k}\right|$, from (2.4) one deduces $\left\|S_{h}\right\|_{h} \leq\left\|S_{h}^{0}\right\|_{h}+T \cdot\left\|\phi_{h}\right\|_{h}+\left\|S_{L_{i}}\right\|_{h}$.

It mean that the finite difference scheme $(2.1)$ is stable unconditionally with respect to $\tau / \Delta$. 
3. Calculation of the matter propagation on a river and the nonnegative property of the numerical solution

Let the function $\phi(x, t)$, boundary condition and initial condition $S^{0}(x)$ be nonnegative. We shall prove that the matter concentration $S_{n}^{k}$ on a river is non-negative. For this we suppose that $S_{n}^{k} \geq 0$ and prove $S_{n}^{k+1} \geq 0$.

1) At the left boundary $L_{1}$ :

i) We have the given boundary condition $S_{L_{i}}(t)=f_{1}(t) \geq 0$ if the flow is inflow (i.e. $v_{1} \geq 0$ ), therefore

$$
S_{1}^{k+1}=f_{1}\left(t_{k+1}\right) \geq 0 .
$$

ii) If the flow is outflow $\left(v_{1}<0\right)$ we determine the value $S$ as follows: Suppose that there are no source, no process of matter diffusion, creation, decay at the boundary, then we obtain the equation

$$
\frac{\partial S}{\partial t}+v_{1} \frac{\partial S}{\partial s}=0
$$

Equation (3.1) can be solved by the following two methods:

- Method of characteristics (see fig. 2)

* Determine the coordinate of the point A: $x_{A}=x_{1}-v_{1} \tau$

* Determine the index $n$ so that: $x_{n-1}<x_{A}<x_{n}$

* Calculate value $S_{A}^{k}$ by linear interpolation

$$
S_{A}^{k}=S_{n-1}^{k}+\frac{x_{A}-x_{n-1}}{x_{n}-x_{n-1}}\left(S_{n}^{k}-S_{n-1}^{k}\right) .
$$

* Take $S_{1}^{k+1}=S_{A}^{k}$

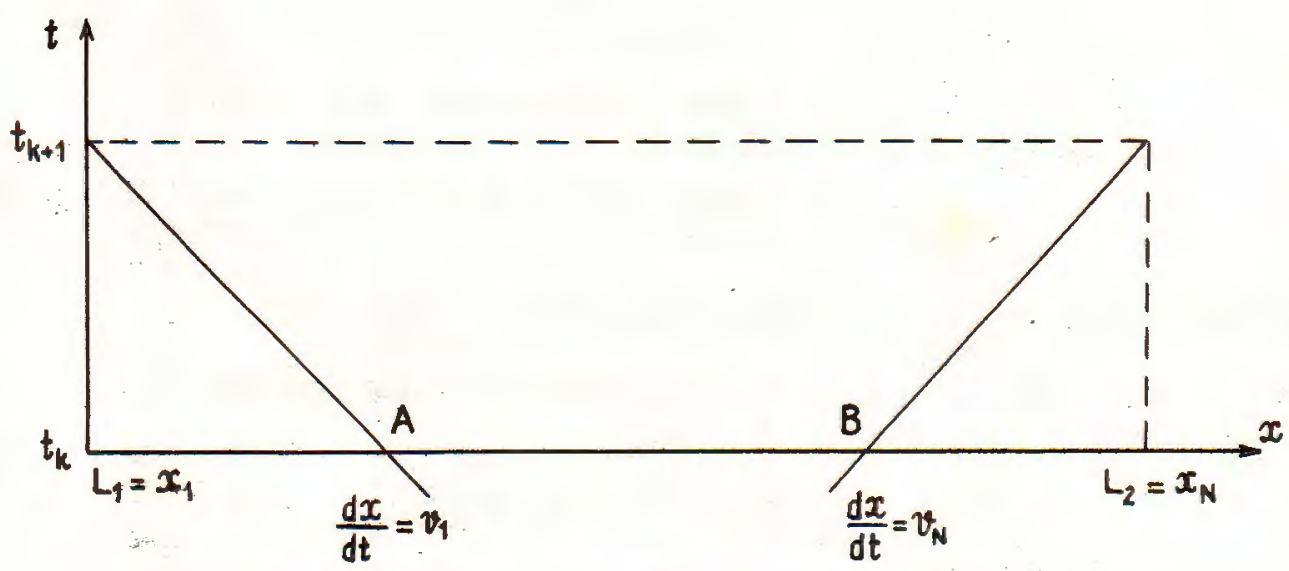

Fig. 2 
- Difference method:

* Determine the least index $n$ so that

$$
\begin{gathered}
x_{n}-x_{1}>\tau\left|v_{1}\right|, \\
\frac{S_{1}^{k+1}-S_{1}^{k}}{\tau}+v_{1} \frac{S_{n}^{k}-S_{1}^{k}}{x_{n}-x_{1}}=0, \\
S_{1}^{k+1}=\left(1-\frac{\tau\left|v_{1}\right|}{x_{n}-x_{1}}\right) S_{1}^{k}+\frac{\tau\left|v_{1}\right|}{x_{n}-x_{1}} S_{n}^{k} .
\end{gathered}
$$

Obviously by both methods we get $S_{1}^{k+1} \geq 0$ if $S_{n}^{k} \geq 0$.

2) At the right boundary $L_{2}$ :

i) We have also the given boundary condition $S_{L_{2}}(t)=f_{2}(t) \geq 0$ if the flow is inflow $\left(v_{N} \leq 0\right)$. Therefore

$$
S_{N}^{k+1}=f_{2}\left(t_{k+1}\right) \geq 0 .
$$

ii) If the flow is outflow $\left(v_{N}>0\right)$ the value $S_{N}^{k+1}$ may be calculated also from the equation

$$
\frac{\partial S}{\partial t}+v_{N} \frac{\partial S}{\partial x}=0
$$

by two methods:

- Method of characteristics (see Fig. 2)

$$
S_{N}^{k+1}=S_{B}^{k}
$$

- Difference method

$$
S_{N}^{k+1}=\left(1-\frac{\tau v_{N}}{x_{N}-x_{n}}\right) S_{N}^{k}+\frac{\tau v_{N}}{x_{N}-x_{n}} S_{n}^{k}
$$

where the maximum index $n$ is chosen so that $\tau v_{N}<x_{N}-x_{n}$. We get also $S_{N}^{k+1} \geq 0$ if $S_{n}^{k} \geqq 0$,

3) At the interior points $x_{n}(n=2,3, \ldots, N-1)$

Equation system (2.2) with the coefficients satisfying the condition (2.3) has the unique solution (see [1]).

System (2.2) is solved by the double sweep method

$$
S_{n+1}^{k+1}=\mathcal{L}_{n+1} S_{n}^{k+1}+r_{n+1},
$$

where

$$
\begin{array}{ll}
\mathcal{L}_{n+1}=\frac{-\gamma_{n+1}}{\beta_{n+1}+\alpha_{n+1} \mathcal{L}_{n+2}} ; \quad \mathcal{L}_{N}=0, \\
r_{n+1}=\frac{\delta_{n+1}-\alpha_{n+1} r_{n+2}}{\beta_{n+1}+\alpha_{n+1} \mathcal{L}_{n+2}} ; \quad r_{N}=S_{N}^{k+1}
\end{array}
$$


and the computational error is not accumulated (see [2], [3]). From (3.6) we have

$$
\mathcal{L}_{N}=0 \text { and } \quad r_{N}=S_{N}^{k+1} \geq 0 .
$$

Using inductive method we can prove that

$$
0<\mathcal{L}_{n}<1 \text { and } r_{n} \geq 0 \quad(n=2, \ldots, N-1) .
$$

Indeed, suppose that

$$
0 \leq \mathcal{L}_{n+1}<1 \text { and } r_{n+1} \geq 0 .
$$

From (2.2), (2.3) and $\phi(x, t) \geq 0$ it yields $\delta_{n} \geq 0$ and

$$
\begin{aligned}
\beta_{n}+\alpha_{n} \mathcal{L}_{n+1} & =\beta_{n}-\left|\gamma_{n}\right|-\left|\alpha_{n}\right|+\left|\gamma_{n}\right|+\left|\alpha_{n}\right|-\left|\alpha_{n}\right| \mathcal{L}_{n+1} \\
& =\sigma_{n}+\left|\gamma_{n}\right|+\left|\alpha_{n}\right|\left(1-\mathcal{L}_{n+1}\right)>\left|\gamma_{n}\right|
\end{aligned}
$$

therefore

$$
\begin{aligned}
0 & \leq \mathcal{L}_{n}=\frac{-\gamma_{n}}{\beta_{n}+\alpha_{n} \mathcal{L}_{n+1}}=\frac{\left|\gamma_{n}\right|}{\beta_{n}+\alpha_{n} \mathcal{L}_{n+1}}<\frac{\left|\gamma_{n}\right|}{\left|\gamma_{n}\right|}=1, \\
r_{n} & =\frac{\delta_{n}-\alpha_{n} r_{n+1}}{\beta_{n}+\alpha_{n} \mathcal{L}_{n+1}}=\frac{\delta_{n}+\left|\alpha_{n}\right| r_{n+1}}{\beta_{n}+\alpha_{n} \mathcal{L}_{n+1}} \geq 0
\end{aligned}
$$

From (3.7) and (3.6) we get

$$
S_{n+1}^{k+1}=\mathcal{L}_{n+1} S_{n}^{k+1}+r_{n+1} \geq 0 .
$$

If the boundary condition at the outflow boundary $L_{i}$ is $\left.\frac{\partial S}{\partial x}\right|_{L_{i}}=0$, then from this boundary condition and (3.6) one deduces:

a) For the case $L_{2}$ is outflow and $L_{1}$ is inflow boundaries:

$$
\mathcal{L}_{N}=1, \quad r_{N}=0 \quad \text { and } \quad S_{1}^{k+1}=f_{1}\left(t_{k+1}\right) \geq 0 .
$$

b) For the case $L_{1}$ is outflow and $L_{2}$ is inflow boundaries:

$$
\mathcal{L}_{N}=0, \quad r_{N}=f_{2}\left(t_{k+1}\right) \quad \text { and } \quad S_{1}^{k+1}=\frac{r_{2}}{1-\mathcal{L}_{2}} \geq 0
$$

Conclusion. Any finite difference scheme with the coefficients $\alpha_{n}, \beta_{n}, \gamma_{n}$ of the equation (2.2) satisfying the condition (2.3) is unconditional stable and the numerical solution is not negative. 


\section{Calculation of the matter propagation on a liver system}

We divide the nodes of a river system into two kinds: the boundary nodes and the internal nodes.

1) At the boundary node $A, B, C$ (see Fig. 1)

The value $S_{1_{i}}^{k+1}$ or $S_{N_{i}}^{k+1}$ is calculated by the formulas (3.2), (3.3) or (3.4), (3.5), where $i \in I, I$ is the set of all boundary nodes.

2) At the internal nodes $D, E, F$

We shall calculate simultaneously the values $S_{j}^{k+1}$ of the all internal nodes $j \in J$, $J$ is the set of internal nodes.

Differencing the adjoint condition (0.2) at the node $D$, we get

$$
\sum_{i=1}^{I^{D}}\left(\frac{\omega P}{\Delta}\right)_{i}^{D}\left(S^{D}-S_{i}^{*}\right)=0
$$

where $S_{i}^{*}=S_{m, i}^{k+1}$

$$
m= \begin{cases}2 & \text { if } D \text { is left boundary of branch } i \\ N-1 & \text { if } D \text { is right boundary of branch } i\end{cases}
$$

3) At the interior points of the river branch $i$ the values $S_{n_{i}}^{k+1}$ are calculated by the following double sweep method

$$
S_{n+1}^{k+1}=\widetilde{\mathcal{L}}_{n+1} S_{n}^{k+1}+\widetilde{r}_{n+1} S_{N}^{k+1}+R_{n+1},
$$

where

$$
\begin{array}{ll}
\widetilde{\mathcal{L}}_{n+1}=\frac{-\gamma_{n+1}}{\beta_{n+1}+\alpha_{n+1} \widetilde{\mathcal{L}}_{n+2}} ; & \widetilde{\mathcal{L}}_{N}=0, \\
\widetilde{r}_{n+1}=\frac{-\alpha_{n+1} \widetilde{r}_{n+2}}{\beta_{n+1}+\alpha_{n+1} \widetilde{\mathcal{L}}_{n+2}} ; & \widetilde{r}_{N}=1, \\
R_{n+1}=\frac{\delta_{n+1}-\alpha_{n+1} R_{n+2}}{\beta_{n+1}+\alpha_{n+1} \widetilde{\mathcal{L}}_{n+2}} ; & R_{N}=0 .
\end{array}
$$

From (4.2) with $n=1$ it yields

$$
S_{2}^{k+1}=\widetilde{\mathcal{L}}_{2} S_{1}^{k+1}+\widetilde{r}_{2} S_{N}^{k+1}+R_{2}=\nu_{2} S_{1}^{k+1}+\mu_{2} S_{N}^{k+1}+\theta_{2},
$$

where $\nu_{2}=\widetilde{\mathcal{L}}_{2}, \mu_{2}=\widetilde{r}_{2}, \theta_{2}=R_{2}$. We have in general

$$
S_{n}^{k+1}=\nu_{n} S_{1}^{k+1}+\mu_{n} S_{N}^{k+1}+\theta_{n},
$$


where

$$
\begin{aligned}
\nu_{n} & =\widetilde{\mathcal{L}}_{n} \nu_{n-1} ; & & \nu_{1}=1, \\
\mu_{n} & =\widetilde{r}_{n}+\widetilde{\mathcal{L}}_{n} \mu_{n-1} ; & & \mu_{1}=0 \\
\theta_{n} & =R_{n}+\widetilde{\mathcal{L}}_{n} \theta_{n-1} ; & & \theta_{1}=0 .
\end{aligned}
$$

From (4.3) one deduces

$$
S_{m, i}^{k+1}=\nu_{m, i} S_{1, i}^{k+1}+\mu_{m, i} S_{N, i}^{k+1}+\theta_{m, i}
$$

Putting (4.4) into (4.1) we get

$$
\sum_{i=1}^{I^{D}}\left(\frac{\omega P}{\Delta}\right)_{i}^{D}\left(S^{D}-\nu_{m, i} S_{1, i}^{k+1}-\mu_{m, i} S_{N, i}^{k+1}-\theta_{m, i}\right)=0 .
$$

One of the values $S_{1, i}^{k+1}$ or $S_{N, i}^{k+1}$ will be matter concentration $S$ at the node $D$ (it depends on that $D$ is left or right boundary of river branch $i$ ). The other one will be concentration $S$ at the second boundary of river branch $i$ (for example at boundary node $A$, or internal node $E$, or internal node $F$ ).

Equation (4.5) is a linear equation describing the correlation of the matter concentrations $S$ at $\left(I^{D}+1\right)$ nodes of the river system.

At each internal node there is always one linear equation like (4.5). Therefore we get the linear algebraic equation system:

$$
A S=c
$$

having the order equal to the number of internal modes and the unknowns are the matter concentrations $S$ at the mentioned nodes. Solving the equation system (4.6) we get the matter concentrations $S$ at all of the internal nodes of the river system.

4) At the interior point $x_{n}$ of each river branch $i$

We shall calculate the values $S_{n, i}^{k+1}(n=2,3, \ldots, N-1)$ by the formula (4.2) or (4.3)' or (3.6).

Remark. At each internal node (for example, node $D$ ), the values $S_{D_{i}}^{k+1}$ may be calculated separately. We have two methods for calculating these values.

1. From the adjoint condition (0.2) one deduces

$$
S_{D_{i}}^{k+1}=S_{D}^{k+1}=\frac{\sum_{i=1}^{I^{D}}\left(\frac{\omega P}{\Delta}\right)_{i} S_{i}^{*}}{\sum_{i=1}^{I^{D}}\left(\frac{\omega P}{\Delta}\right)_{i}}
$$


where

$$
S_{i}^{*}= \begin{cases}S_{2, i}^{k+1}, & \text { if } D \text { is left boundary of river branch } i \\ S_{N-1, i}^{k+1}, & \text { if } D \text { is right boundary of river branch } i\end{cases}
$$

and $S_{2, i}^{k+1}, S_{N-1, i}^{k+1}$ may be determine by the method of characteristic or explicit finite difference method.

2) Using other adjoint condition (see [7], [8]), we have

$$
S_{D_{j}}^{k+1}=\frac{\sum_{i \in I_{v}^{D}}\left|Q_{D_{i}}\right| S_{D_{i}}^{k+1}}{\sum_{j \in I_{r}^{D}}\left|Q_{D_{j}}\right|}, \quad j \in I_{r}^{D},
$$

where $I_{v}^{D}$ is the set of river branches having inflow at the node $D, I_{r}^{D}$ - the set of branch having outflow at $D, I^{D}=I_{v}^{D}+I_{r}^{D}, Q_{D_{i}}$ is the water discharge of branch $i$ at $D$.

The values $S_{D_{i}}^{k+1}, i \in I_{v}^{D}$ are calculated by the method of characteristic.

The above algorithm was used for calculating the matter propagation in the DONGNAI-SAIGON river system (see [5]), where $v$ is computed from the one dimensional Saint-Venant equation system (see [6]):

$$
\begin{aligned}
& B \frac{\partial Z}{\partial t}+\frac{\partial Q}{\partial x}=q, \\
& \frac{\partial Q}{\partial t}+2 v \frac{\partial Q}{\partial x}+B\left(c^{2}-v^{2}\right) \frac{\partial Z}{\partial x}=\left[i B+\left(\frac{\partial \omega}{\partial x}\right)_{h}\right] v^{2}-\frac{g \omega Q|Q|}{\mathcal{K}^{2}}
\end{aligned}
$$

by using the difference scheme

$$
\begin{aligned}
& \left.\frac{\partial f}{\partial t}\right|_{n+1 / 2} ^{k+1}=\frac{f_{n+1}^{k+1}+f_{n}^{k+1}-f_{n+1}^{k}-f_{n}^{k}}{2 \tau}, \\
& \left.\frac{\partial f}{\partial x}\right|_{n+1 / 2} ^{k+1}=\frac{f_{n+1}^{k+1}-f_{n}^{k+1}}{\Delta} .
\end{aligned}
$$

And the DONGNAI-SAIGON river system was modelled as follows

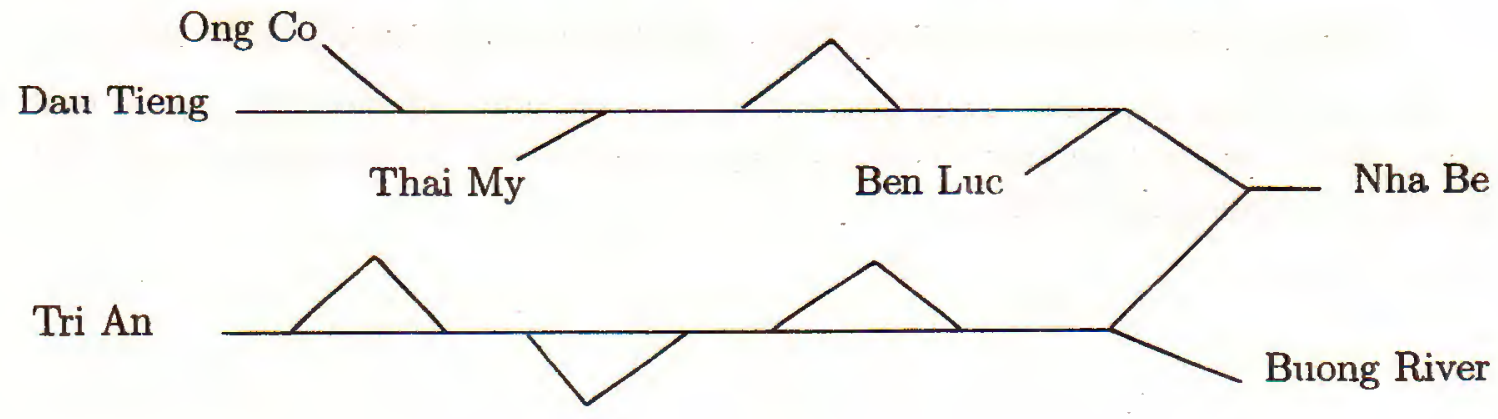


This work is partially supported by the Council for Natural Sciences of Vietnam and by the Program "Applied Mathematics" NCNST.

\section{REFERENCES}

1. Godunov S. K. Difference scheme, Publishers "Nauka", Moscow, 1977.

2. Roach P. J. Computational Aluid dynamics, Hermosa Publishers, Albuquerque, 1976.

3. Richtmyer R. D., Morton K. W. Difference methods for initial-value problems, second edition, Interscience Publishers, New York-London-Sydney, 1967.

4. Lions J., Megenes E. Problèmes aux limites non homogènes et applications, T. 1, Paris, Dunod 1968.

5. Nguyen Van Gia, Tran Van Lich, Pham Van Ninh. One dimensional hydraulic and diffusion problems in the forecast of water pollution for systems of rivers and open channels (Scientific report of the contract with the Department for design of TRI-AN hydroelectric station), Hanoi, 1985, 31 pages (in Vietnamese).

6. Vasiliev O. F., Temnoeva T. A., Sugrin S. M. Numerical methods for calculating the unsteady flow on the open channel (Proceedings AN SSSR - Mechanics), No2, 1965, 17-25.

7. Tran Gia Lich, Nguyen Cong Dieu. Numerical method for solving the diffusion problems in the river and open channel system, Working paper, Institute of Mathematics, Hanoi 1989.

8. Nguyen Tat Dac, Nguyen Van Diep. Calculation of tidal propagation and sanility intruction in river system by one-dimensional mathematical models. International symposium on the salinity intruction in the Mekong Delta, Ho Chi Minh City, 1982, 97-126 (in Vitnamese).

Received October 4, 2000

TÍNH LAN TRUYÈ̀ VẬT CHẨT TRÈN HỆ THỐNG SÔNG HOẶC KÊNH HỞ

Bài báo trình bày các vấn đề sau: Sự tồn tại nghiệm của bài toán, sự ổn định của sơ đồ sai phân, tính chất không âm của nghiệm bằng số khi các điều kiện đầu và diều kiện biên không âm. 